\title{
Interrupção da gestação após o diagnóstico de malformação fetal letal: aspectos emocionais
}

\author{
Termination of pregnancy after the diagnosis of lethal fetal malformation: emotional aspects
}

Gláucia Rosana Guerra Benute ${ }^{1}$, Roseli Mieko Yamamoto Nomura², Mara Cristina Souza de Lucia ${ }^{3}$, Marcelo Zugaib ${ }^{4}$

\section{RESUMO}

\begin{abstract}
Objetivos: descrever os processos emocionais vivenciados com a interrupção da gestação após o diagnóstico de malformação fetal letal. Métodos: foram entrevistadas 35 gestantes cujo feto era portador de malformação letal e que realizaram a interrupção da gestação após solicitação de autorização judicial. A malformação fetal mais freqüente foi a anencefalia (71,5\%). As pacientes foram submetidas à entrevista psicológica aberta logo após o diagnóstico da malformação fetal para que pudessem expressar os sentimentos desencadeados e para promover reflexão sobre a solicitação da interrupção da gravidez. O tempo médio de espera pelo deferimento do pedido judicial foi de 16,6 dias. As que solicitaram e tiveram o aborto realizado foram convidadas a retornar para a segunda entrevista psicológica 30 a 60 dias após o procedimento, quando foi aplicado questionário semidirigido para identificar os aspectos emocionais vivenciados e descrever os sentimentos despertados. Resultados: trinta e cinco pacientes foram entrevistadas após o aborto. Quanto aos sentimentos vivenciados na decisão pela interrupção, $60 \%$ relataram como negativos, $51,4 \%$ afirmaram que não tiveram dúvidas quanto à decisão tomada e $65,7 \%$ informaram que a própria opinião foi a que mais pesou na decisão. A maioria das mulheres (89\%) afirmou apresentar lembranças do que viveram com certa freqüência, $91 \%$ afirmou que adotariam a mesma atitude em outra situação semelhante e 60\% diriam para interromper a gestação caso alguém perguntasse seu conselho, numa mesma situação. Conclusões: as angústias vivenciadas demonstram que o processo de reflexão é de fundamental importância para decisão consciente e posterior satisfação com a atitude tomada. $\mathrm{O}$ acompanhamento psicológico permite revisão dos valores morais e culturais para auxiliar a tomada de decisões visando minimizar o sofrimento vivido.
\end{abstract}

PALAVRAS-CHAVE: Feto/anormalidades; Aborto legal; Complicações da gravidez; Anencefalia; Entrevistas

\section{ABSTRACT}

Purpose: to describe to emotional process experienced with termination of pregnancy after the diagnosis of lethal fetal malformation. Methods: thirty-five pregnant women who underwent termination of pregnancy for lethal fetal anomaly after judicial permission were interviewed. The most frequent fetal malformation was anencephaly $(71.5 \%)$. The patients were submitted to an open interview as soon as the diagnosis of fetal malformation was confirmed, allowing them to express their feelings and stimulating them to think about asking for termination of pregnancy. The mean time spent until the judicial agreement was 16.6 days. The women who requested and were submitted to the procedure of abortion were invited to return for psychological evaluation after 30-60 days. At this moment, a semi-structured interview was performed to find the emotional aspects and feelings that existed. Results: thirty-five patients were interviewed. The decision-making feelings about termination of pregnancy were negative for $60 \%, 51.4 \%$ declared that they had no doubts about the assumed decision and $65.7 \%$ declared that their own opinion was more important for decision than anyone else's. Most of the women (89\%) affirmed to remember the facts about the procedure that they experienced, $91 \%$ affirmed that they would have the same attitude in the case of another similar situation in the future and $60 \%$ declared that they would advise someone to opt for termination of pregnancy if asked about the same situation. Conclusions: the anguish experienced showed that the process

Clínica Obstétrica do Hospital das Clínicas da Faculdade de Medicina da Universidade de São Paulo - USP - São Paulo (SP) - Brasil.

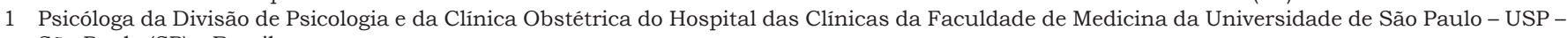
São Paulo (SP) - Brasil.

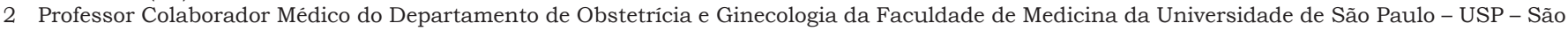
Paulo (SP) - Brasil.

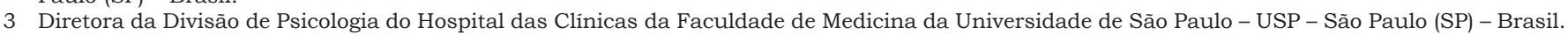

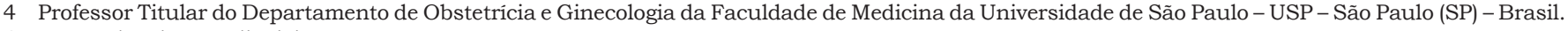

Correspondência: Roseli Mieko Yamamoto Nomura

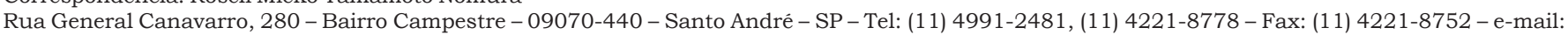
roseli.nomura@terra.com.br 
of thinking is very important for the decision-making process and posterior satisfaction with the assumed posture. The psychological follow-up allows to review the moral and cultural values in order to help the decision-making process with the aim of minimizing the suffering.

KEYWORDS: Fetus/abnormalities; Abortion, legal; Pregnancy complications; Anencephaly; Interview

\section{Introdução}

A Medicina Fetal, compreendida como parte integrante da obstetrícia, objetiva avaliar a saúde e a vitalidade fetal. Tem como princípio fornecer informações sobre diagnósticos e prognósticos fetais, indicando e orientando a paciente sobre a melhor forma de atuação de acordo com cada situação.

$\mathrm{O}$ avanço tecnológico possibilitou a introdução de novos métodos na propedêutica fetal. A ultrasonografia obstétrica passou a ser utilizada nos países desenvolvidos, na década de 1950, chegando ao Brasil nos anos 70, com a ultra-sonografia para fins diagnósticos ${ }^{1}$. Atualmente, com o aprimoramento das técnicas e dos equipamentos, é possivel a confirmação de muitas hipóteses diagnósticas sobre a condição fetal, colocando os pais em contato direto com uma realidade cujo acesso só seria possível após o nascimento ${ }^{2-4}$.

Grande número de gestantes são submetidas ao exame de ultra-sonografia obstétrica. Geralmente, este método propedêutico de diagnóstico pré-natal objetiva obter informações que confirmem a saúde do feto, eliminando ou minimizando os medos e anseios da existência de malformações fetais ${ }^{5}$. Entretanto, eventualmente, diagnósticos desfavoráveis são estabelecidos quando são detectadas anomalias no produto conceptual.

O diagnóstico pré-natal é evento estressante, na medida em que existe a possibilidade do diagnóstico de anomalias fetais ${ }^{6}$. O enfrentamento da realidade nem sempre é processo de fácil elaboração, principalmente quando a normalidade não é diagnosticada. São observados sinais de morbidade psicológica e sintomas de estresse pós-traumático após a interrupção da gestação por anomalias fetais ${ }^{7}$. E ainda, quando se presume que a anomalia fetal seja compativel com a vida, sinais de sofrimento e dúvidas são freqüentemente relatados, principalmente devido aos questionamentos acerca da qualidade de vida e do prognóstico a longo prazo $^{8}$. Sintomas de estresse pós-traumático também são verificados em maior intensidade quando a interrupção é realizada em idade gestacional avançada, a partir do segundo trimestre da gravidez $z^{8}$
Ao informar o diagnóstico de malformação fetal, espera-se que o auxílio à paciente se caracterize pela neutralidade no esclarecimento sobre os procedimentos diagnósticos e terapêuticos. Assim, a imparcialidade do profissional é de importância fundamental para que o casal possa se remeter às suas próprias crenças e convicções, desencadeando processo de reflexão que poderá auxiliar se eventualmente for discutida a interrupção ou não da gestação ${ }^{9}$.

Mas a grande questão persiste na limitação atual encontrada pelos profissionais que trabalham com Medicina Fetal. O que fazer após obter um diagnóstico de malformação fetal letal? Como aconselhar?

A legislação de países da Europa ou da América do Norte permite que o casal escolha entre interromper ou não a gestação, quando o feto está gravemente acometido por afecção incompatível com a vida. Entretanto, no Brasil, o procedimento do aborto não é permitido pelo Código Penal Brasileiro ${ }^{10}$. Do ponto de vista legal, considera-se aborto a interrupção do processo da gravidez, com a morte do feto, independentemente do estágio de desenvolvimento em que se encontre a gestação. Pela legislação vigente, o aborto não é punido quando é necessário para preservar um bem maior: a vida da gestante ou a honra da mulher violentada. Por não haver legislação específica sobre a interrupção da gravidez, nos casos de malformação fetal letal, verifica-se a necessidade de interpretação da lei e de discussão sobre o tema por juristas e pela sociedade em geral ${ }^{11}$.

Portanto, no Brasil, não havendo respaldo legal que autorize o aborto nos casos de malformação fetal letal, o casal que enfrenta este diagnóstico se vê angustiado na busca de soluções para o problema. O acompanhamento psicológico deve necessariamente ser oferecido a esses casais. Ao gerar um filho malformado, os pais, muitas vezes, sentem que o que eles tem de pior foi passado ao filho e agora estão expostos para a sociedade todos os seus erros, todos os seus defeitos ${ }^{12}$. Desse modo, verifica-se que intensas vivências emocionais são desencadeadas a partir do diagnóstico de malformação fetal letal ${ }^{7,13}$.

Atualmente, com o desenvolvimento das técnicas de diagnóstico pré-natal, o feto passou a ser agente ativo, tornando-se o paciente da Medicina 
Fetal. A aplicação de métodos propedêuticos no período antenatal diagnosticando anomalias no concepto propicia a instalação de sofrimento e confli$\operatorname{tos}^{8}$ para a gestante e seus familiares, o que torna necessário o tratamento e profilaxia dos aspectos emocionais por profissional especializado ${ }^{6,9,14}$.

Dentro deste contexto atual, não existem dados nacionais acerca das vivências emocionais de casais que se deparam com o diagnóstico de malformação fetal letal. O presente estudo tem como objetivo identificar os processos psíquicos desencadeados nas mulheres que, diante do diagnóstico de malformação fetal letal, solicitaram a autorização judicial para a interrupção da gravidez. Os conhecimentos gerados a partir desta investigação contribuirão para o aprimoramento de condutas a serem adotadas, facilitando a compreensão do processo psíquico, auxiliando no psicodiagnóstico da situação vivenciada e no planejamento de intervenções.

\section{Métodos}

Foram estudadas prospectivamente pacientes atendidas na Clínica Obstétrica do Hospital das Clínicas da Faculdade de Medicina da Universidade de São Paulo que receberam o diagnóstico de malformação fetal letal, no período de janeiro de 2001 a dezembro de 2003.

No período analisado foram solicitados 81 pedidos de interrupção judicial da gravidez, por anomalia fetal letal. Cinqüenta e três pedidos $(65,4 \%)$ foram deferidos pelo judiciário, que emitiu o alvará de autorização do aborto, quatro pedidos $(4,9 \%)$ foram negados, em quatro casos $(4,9 \%)$ ocorreu o óbito fetal antes da tramitação da solicitação pela interrupção e 20 pacientes $(24,7 \%)$ não retornaram ao serviço.

Nenhuma paciente se recusou expressamente a participar da pesquisa. Das 53 pacientes que foram submetidas à interrupção da gestação, $35(66 \%)$ retornaram para a entrevista psicológica após o procedimento. Como critérios de inclusão foram considerados os seguintes aspectos: paciente que teve o diagnóstico de malformação fetal letal, que solicitou e obteve autorização judicial para a interrupção da gestação, com procedimento para aborto realizado nesta instituição. Somente foram incluídas as pacientes orientadas e esclarecidas sobre a pesquisa na época da internação e que retornaram para a entrevista semidirigida 30 a 60 dias após o procedimento. $\mathrm{O}$ termo de consentimento livre e esclarecido foi solicitado quando a mesma retornou para a ava- liação pós-procedimento, momento em que foi novamente ressaltado a não-obrigatoriedade de participação nesta pesquisa, sua independência com relação à qualidade do atendimento e a necessidade de serem utilizadas as informações colhidas na entrevista. Após a leitura, tendo a mulher concordado expressamente em participar do estudo, foi iniciada a entrevista semidirigida.

A idade materna variou de 13 a 42 anos, apresentando média de 25 anos e desvio padrão (DP) de 6,3 anos. Quanto ao número de gestações, $15(42,9 \%)$ eram primigestas, $10(28,6 \%)$ secundigestas e $10(28,6 \%)$ apresentavam três ou mais gestações anteriores. Em relação ao número de filhos vivos, 48,6\% (17 casos) não apresentavam nenhum filho vivo. A idade gestacional apresentou média de 18 semanas com DP de 5,5 semanas. A investigação dos dados relativos à religião demonstrou que $26(74,3 \%)$ pacientes eram católicas, quatro $(11,4 \%)$ evangélicas, uma $(2,9 \%)$ da religião espírita, uma $(2,9 \%)$ era mórmon e duas não tinham religião $(5,7 \%)$. Quanto ao grau de escolaridade verificou-se que $19(54,3 \%)$ relatavam $1^{\circ}$ grau, $15(42,9 \%)$ tinham $2^{\circ}$ grau e uma, grau superior $(2,9 \%)$. Sobre a relação conjugal, 21 $(60,1 \%)$ coabitavam com o companheiro (casadas ou com união estável).

O tipo de malformação fetal letal detectado pelo diagnóstico ultra-sonográfico ou genético, que indicou a solicitação da interrupção da gestação, está descrito na Tabela 1 . Os casos foram caracterizados conforme a anomalia de maior gravidade. Destaca-se o diagnóstico de anencefalia em 71,5\%.

Tabela 1 - Principais anomalias detectadas nos fetos de 35 gestantes, com diagnóstico de malformação fetal letal, entrevistadas após a interrupção judicial da gravidez

\begin{tabular}{lrr}
\hline Diagnóstico & \multicolumn{2}{c}{ Resultados } \\
\hline Anencefalia & 25 & $71,4 \%$ \\
Malformação nefrourológica & 4 & $11,4 \%$ \\
Sistema nervoso central $^{*}$ & 3 & $8,6 \%$ \\
Síndromes genéticas $_{\text {Malformações múltiplas }}$ & 2 & $5,8 \%$ \\
\hline
\end{tabular}

${ }^{*}$ Foram excluídos os casos de anencefalia.

De acordo com o protocolo assistencial da Clínica Obstétrica, todas as pacientes que receberam o diagnóstico de malformação fetal letal foram inicialmente submetidas à entrevista psicológica aberta, realizada imediatamente após a consulta com o profissional da área de Medicina $\mathrm{Fe}-$ tal, momento em que foi dado ou confirmado o diagnóstico de malformação fetal letal. A realização da entrevista aberta teve como objetivo central propiciar a expressão dos sentimentos desencadeados com o diagnóstico da malformação, promo- 
ver reflexão acerca do desejo da solicitação da interrupção judicial da gravidez e, ainda, a finalidade de permitir que a mulher e/ou casal falassem livremente sobre o que estavam vivendo, as angústias e as aflições decorrentes do diagnóstico. Nesse primeiro encontro, foi realizada a orientação psicológica de acordo com a necessidade específica de cada caso, sempre com o intuito de auxiliar no enfrentamento do problema.

Após essa entrevista aberta, as que desejavam interromper a gravidez seguiram a rotina estabelecida no departamento, pela qual recebem toda a orientação necessária e os laudos para a solicitação do alvará judicial para autorização do aborto.

Após a realização da interrupção da gestação, obedecidos os critérios de inclusão e exclusão, foi realizada a entrevista semidirigida, no mínimo 30 dias e no máximo 60 dias após o aborto. Todas as entrevistas foram realizadas pela mesma pesquisadora. Este tipo de entrevista permitiu identificar os aspectos emocionais vivenciados no processo de decisão pela interrupção judicial da gravidez e descrever os sentimentos despertados a partir desse processo. As questões abordadas foram categorizadas de acordo com o seu conteúdo e conforme o tema central das respostas de cada uma delas.

A opção pela utilização da entrevista semidirigida deu-se em função da necessidade de permitir também que a mulher falasse livremente sobre o tema de cada questão, de forma que houvesse subsídios para a análise dos dados, propiciando melhor conhecimento da vivência emocional.

Para a análise dos dados das entrevistas semidirigidas foi utilizada a técnica de análise temática ou análise de conteúdo, que tem como objetivo permitir a interpretação do discurso, de forma direta, sistemática e quantitativa do conteúdo. Tem a finalidade de descrever, interpretar e compreender os dados. Neste tipo de análise qualitativa é realizada a análise da comunicação, visando obter, por procedimentos sistemáticos e objetivos de descrição do conteúdo das mensagens, indicadores que permitam a inferência de conhecimentos relativos às mensagens que estão contidas nas respostas apresentadas. Procurou-se descobrir, em cada resposta, qual era o núcleo de sentido da mensagem, procurando identificar núcleos cuja presença ou freqüência apresentassem significado para o objetivo analítico visado. Inicialmente procedeu-se a uma categorização das respostas e os dados foram codificados. Dessa forma, as respostas dissertativas da entrevista transformaram-se em categorias por meio de análise transversal, isto é, as entrevistas realizadas foram recortadas ao redor de cada tema-eixo, ou unidade de significação, da questão formulada na entrevista semidirigida, encontrando-se, assim, os núcleos de sentido que compõem a comunicação de cada paciente, codificando os dados do discurso, categorizando os conteúdos.

Todos os dados obtidos com a categorização foram analisados quantitativamente, pois essa análise constrói índices que permitem formas de quantificação. Os resultados encontram-se apresentados em forma de tabelas.

A interpretação das respostas relacionadas aos sentimentos vivenciados durante a decisão da interrupção foi categorizada em: aspectos negativos, segurança e desorientação. A categoria de sentimentos negativos relacionou-se às respostas que indicavam choque, angústia, tristeza, resignação, destruição de planos, revolta, medo, vergonha, inutilidade, choro, incapacidade de ser mãe, indignação e insegurança como mulher. Nos casos categorizados como sentimentos de segurança as respostas apresentavam conteúdo indicando racionalidade da pessoa, imediatismo, conformação com a letalidade da anomalia fetal, inevitabilidade da letalidade, certeza na tomada de decisão pela interrupção, alívio com a opção, possibilidade de encurtar o tempo de sofrimento com a interrupção e decisão consciente. As respostas que foram categorizadas como sentimentos de desorientação envolviam relatos de não-aceitação do diagnóstico, de descrença, vontade de se enganar, dúvidas na tomada de decisões, dúvidas sobre os sentimentos do feto, dificuldade em permanecer com a gravidez e sinais de incerteza.

Para a análise das questões que abordavam as lembranças do período vivenciado na interrupção, os sentimentos relacionados à vivência do processo foram interpretados de forma que denominou-se 'filho imaginário' às respostas que remetiam, de algum modo, à imagem que faziam do filho perfeito, do filho que poderia ter nascido, se não tivesse as anomalias. Por sentimentos negativos foram incluídas as respostas que falavam da tristeza desencadeada com o diagnóstico; na categoria adequada foram incluídas as respostas que falavam da certeza de ter tomado a melhor atitude interrompendo a gravidez e denominaram-se lembranças específicas as respostas que elencavam a realização de um exame em particular, por exemplo, a ultra-sonografia.

Esta pesquisa foi avaliada e aprovada pela Comissão de Ética em Pesquisa do Hospital das Clínicas da Faculdade de Medicina da Universidade de São Paulo (CAPPesq) sob o número 604/03. As pacientes tiveram assegurada a oportunidade de realizar acompanhamento psicológico na Clínica Obstétrica do Hospital das Clínicas da Faculdade de Medicina da Universidade de São Paulo após a pesquisa, caso houvesse interesse, mas tais atendimentos não fizeram parte deste estudo. 


\section{Resultados}

Com relação à opção pela interrupção judicial da gravidez e os resultados relativos às vivências emocionais do processo de decisão em interromper ou não a gestação tem-se que 14 (40\%) resolveram pela interrupção no mesmo dia, 13 demandaram entre um e sete dias $(37,2 \%)$, quatro entre 8 e 15 dias $(11,4 \%)$ e quatro demoraram mais de 15 dias $(11,4 \%)$.

Ao se proceder à análise das questões relacionadas aos sentimentos vivenciados durante o período de decisão para interromper ou não a gestação, $60 \%$ das pacientes relataram aspectos negativos. Quando indagadas sobre como foi a tomada de decisão para solicitar a interrupção judicial da gravidez, sentimentos negativos foram novamente relatados por $57,1 \%$ das mulheres entrevistadas (Tabela 2).

Tabela 2 - Aspectos e sentimentos vivenciados pelas 35 gestantes com diagnóstico de malformação fetal letal durante 0 período de decisão pela interrupção judicial.

\begin{tabular}{|c|c|c|}
\hline Características & $\mathbf{n}$ & $\%$ \\
\hline \multicolumn{3}{|c|}{ Sentimentos durante decisão de interrupção } \\
\hline Aspectos negativos & 21 & 60,0 \\
\hline Segurança & 10 & 28,6 \\
\hline Desorientação & 4 & 11,4 \\
\hline \multicolumn{3}{|c|}{ Tomada de decisão pela interrupção } \\
\hline Sentimentos negativos & 20 & 57,1 \\
\hline Pragmática & 15 & 42,9 \\
\hline \multicolumn{3}{|l|}{ Dúvidas vivenciadas } \\
\hline Não teve dúvida & 18 & 51,4 \\
\hline Dúvida do diagnóstico & 11 & 31,4 \\
\hline Dúvida moral & 5 & 14,3 \\
\hline Negação & 1 & 2,9 \\
\hline \multicolumn{3}{|c|}{ Incômodo em falar da gravidez } \\
\hline Não & 21 & 60,0 \\
\hline $\operatorname{Sim}$ & 14 & 40,0 \\
\hline \multicolumn{3}{|l|}{ Opinião mais importante } \\
\hline A própria & 23 & 65,7 \\
\hline Marido & 4 & 11,4 \\
\hline Própria e do marido & 4 & 11,4 \\
\hline Outros & 2 & 5,8 \\
\hline \multicolumn{3}{|c|}{ Intervalo de tempo até decisão judicial } \\
\hline Mesmo dia & 3 & 8,6 \\
\hline 1 a 7 dias & 6 & 17,1 \\
\hline 8 a 15 dias & 13 & 37,1 \\
\hline 16 a 30 dias & 10 & 28,6 \\
\hline 30 a 60 dias & 3 & 8,6 \\
\hline
\end{tabular}

Quanto à existência ou não de dúvidas para interromper a gestação, cerca de metade afirmaram que não tiveram dúvidas sobre a decisão, conforme os dados da Tabela 3. A maioria das mulheres $(60 \%)$ afirmou que certo tempo após a interrupção, não se importava em falar da gravidez, do bebê ou do procedimento realizado. Dos motivos sobre o incômodo de falar da interrupção da gravidez, 42,9\% não conseguiu explicar esse aspecto abordado.

Tabela 3 - Sentimentos vivenciados pelas 35 gestantes com diagnóstico de malformação fetal letal após a interrupção judicial.

\begin{tabular}{lrr}
\hline Características & $\mathbf{n}$ & $\%$ \\
\hline Lembranças sobre o período vivenciado na interrupção & & \\
Filho imaginário & 11 & 31,4 \\
Sentimentos negativos & 11 & 31,4 \\
Atitude adequada & 6 & 17,1 \\
Lembranças específicas & 2 & 5,7 \\
Não sabe explicar & 1 & 2,9 \\
Não tem lembranças & 4 & 11,4 \\
O que dizer para alguém na mesma situação & & \\
Interromper & 21 & 60,0 \\
Decisão pessoal & 10 & 28,6 \\
Ambivalente & 2 & 5,7 \\
Não sabe & 1 & 2,9 \\
Não interromper & 1 & 2,9 \\
Alterações no relacionamento conjugal & & \\
Não teve alterações & 22 & 62,9 \\
Melhora no relacionamento & 10 & 28,6 \\
Dificuldade no relacionamento sexual & 1 & 2,9 \\
Ausência de relacionamento estável & 1 & 2,9 \\
Ambivalente & 1 & 2,9 \\
Crença sobre o aborto antes da interrupção judicial & & \\
Contra & 16 & 45,7 \\
Depende & 8 & 22,9 \\
A favor & 6 & 17,1 \\
Não explica & 4 & 11,4 \\
Nunca tinha pensado & 1 & 2,9 \\
Atitude a ser adotada em outra situação semelhante & \\
Sim & 32 & 91,4 \\
Não & 2 & 5,7 \\
Não sabe & 1 & 2,9 \\
Crença em receber algum castigo & & \\
Não & 32 & 91,4 \\
Sim & 2 & 5,7 \\
Não respondeu & 1 & 2,9 \\
\hline & & \\
& &
\end{tabular}

Observa-se que, quanto à opinião que mostrou ser mais importante na tomada da decisão de interromper ou não a gestação, $65,7 \%$ das mulheres afirmaram que a própria opinião foi a mais importante. Quanto aos dados relativos ao tempo de espera até o deferimento do alvará judicial, a média de tempo observada foi de 16,6 dias. Esperaram de 8 a 15 dias $37,1 \%$ dos casos.

As mulheres foram abordadas com relação aos sentimentos pertinentes à vivência da interrupção judicial da gravidez. Os dados relativos à existência ou não de lembranças do período vivido demonstraram que a maioria das mulheres (89\%) afirmou lembrar do que viveram com certa freqüência. Parte das gestantes $(35,5 \%)$ relatou lembranças quanto ao filho imaginário e a mesma proporção de mulheres descreveu sentimentos negativos, conforme a Tabela 3 . 
Quanto a aconselhar outras mães que estivessem indecisas sobre interromper ou não a gestação, nas mesmas condições que a vivida, a maioria das mulheres entrevistadas (60\%) afirmou que aconselhariam a interrupção, conforme os resultados apresentados na Tabela 3.

Solicitou-se que as mulheres pensassem a respeito da existência ou não de alterações no relacionamento conjugal após a interrupção. A maioria, 62,9\%, afirmou que não ocorreram mudanças no relacionamento. Entre as que relataram mudanças, 10 mulheres afirmaram que o relacionamento melhorou (Tabela 3).

Procurou-se compreender qual o sentimento que as mulheres tinham em relação à interrupção voluntária da gravidez, antes e depois da vivência de todo o processo. Buscou-se também avaliar o imaginário das pacientes no que diz respeito à existência ou não de culpa pelo ato realizado. Essa evidência pode ser encontrada nas respostas referentes à atitude em uma situação semelhante: interromperia ou não a gestação, quais os motivos que levariam à interrupção da gestação em outra situação semelhante à vivida, quem soube da interrupção judicial da gravidez, se houve críticas à decisão de interromper a gestação ou críticas diante da decisão do aborto judicial e a crença em castigo no futuro. Observou-se que quanto às crenças relativas ao aborto, antes da vivência da malformação fetal e da interrupção judicial da gravidez, 45,7\% era contra o aborto (Tabela 3).

Quanto à atitude a ser tomada em situação semelhante, a maioria das mulheres (91\%) relatou que agiria da mesma forma, interrompendo a gestação (Tabela 3). Ao serem questionadas se acreditavam que poderiam receber algum tipo de "castigo" no futuro a partir da realização do aborto, a grande maioria das entrevistadas $(97,1 \%)$ relatou que não acreditava nessa possibilidade (Tabela 3).

\section{Discussão}

O processo de internação hospitalar, a indução e interrupção da gestação são situações que envolvem sofrimento pessoal importante. A internação em enfermaria obstétrica propicia contato com gestações que terminaram em sucesso, o que desperta sentimentos mais intensos de fracasso e frustração, não pela interrupção em si, mas pela incapacidade sentida no gestar, pela ausência do filho imaginado, pelo filho perdido.

A reação dos casais em face do diagnóstico pré-natal de uma anormalidade fetal pode envol- ver sentimentos de raiva, desespero, inadequação e distúrbios do sono e de alimentação ${ }^{15}$. Ainda com relação ao diagnóstico de malformação fetal, as reações emocionais desencadeadas, tais como dor, angústia e sofrimento, podem não estar relacionadas com o número de filhos vivos nem com o planejamento e o desejo pela gestação, pois são comuns a todas as mulheres que passam por essa experiência ${ }^{16}$. Com relação à equipe médica, a raiva pode ser dirigida à equipe de saúde, demonstrando que a compreensão é fundamental para auxiliar o casal a se adaptar à nova realidade.

No presente estudo, a maioria das mulheres relatou aspectos negativos vivenciados durante a decisão pela interrupção da gestação. Em estudos que avaliam a incidência de depressão após a interrupção da gestação no segundo trimestre por anomalias fetais, não se observa diferença entre as pacientes que realizaram a indução do parto ou a dilatação e curetagem, quando da realização do procedimento. Entretanto, em ambos os grupos, mais da metade das pacientes apresentaram sintomas depressivos ${ }^{13}$.

Ao receber o diagnóstico de malformação fetal, é esperado que as gestantes passem por períodos de dúvidas e questionamentos, buscando outras opiniões, para confirmar o diagnóstico recebido $^{17}$. Esses sentimentos comumente dizem respeito à ansiedade e apreensão. Quando o diagnóstico é estabelecido com a gestação avançada, a aceitação do problema torna-se muito dificil. O diagnóstico de aneuploidias fetais no período antenatal pode trazer beneficios mesmo para as mulheres que pretendem prosseguir com a gravidez, pois possibilita a busca por informações sobre os cuidados para com o bebê, principalmente programando-se visitas a geneticistas e pediatras, antes do nascimento ${ }^{18}$.

Em países onde o aborto é permitido, quando é estabelecido o diagnóstico de anomalia cromossômica do feto, a opção pela interrupção da gravidez ocorre em elevada percentagem dos casos: $100 \%$ dos casos na Suíça e 94 a 100\% nos Estados Uni$\operatorname{dos}^{19}$. Quando se confirma o diagnóstico de distúrbios metabólicos, $100 \%$ das pacientes realizam a interrupção da gravidez na Austrália, o mesmo ocorrendo nos Estados Unidos. A detecção de espinha bífida leva ao aborto em $100 \%$ das gestações, na Inglaterra e nos Estados Unidos, e em 95\% das vezes na Austrália. Discute-se que a opção pela interrupção da gravidez, muitas vezes, está pautada na qualidade de vida do feto, com relatos de preocupação com o sofrimento do bebê e da família ${ }^{19}$.

Neste estudo foi observado que apenas 51,4\% das mulheres não relatam dúvidas sobre as atitudes tomadas no processo da interrupção judicial 
da gravidez por malformação fetal letal e 43\% assumem a tomada de decisões como ação pragmática de enfrentamento da realidade. Entretanto, as dúvidas vivenciadas pelas pacientes relacionaram-se principalmente ao diagnóstico. Menor proporção relatou dúvidas de ordem moral em relação ao aborto. Em estudo realizado nos Estados Unidos, a opção pela manutenção ou não da gravidez, nos casos de fetos acometidos por alguma anormalidade, parece estar relacionada com a perspectiva da qualidade de vida da criança, da família e o compromisso pessoal ${ }^{20}$.

Em levantamento europeu envolvendo 17 centros com registros de malformações congênitas fetais, verifica-se que $43 \%$ dos casos evoluem para a interrupção da gestação. Nesse estudo constata-se variação entre as diferentes regiões estudadas (15 a 59\%) ${ }^{21}$. Em estudo realizado na China investigando as atitudes das mulheres sobre a interrupção da gestação por meio de questionário estruturado, verifica-se que metade delas compreendem ser direito da mulher a escolha pelo aborto no início da gravidez e, a grande maioria (90\%) optaria pela interrupção da gestação quando na presença de anormalidades cromossômicas letais ou da sindrome de Down ${ }^{22}$.

A internação e a indução do parto são concebidas com juízo de tempo diferente do real. As horas parecem dias, os dias, anos. O desejo de que tudo acabe e o medo de como será depois propicia essa alteração. Os casais conseguem, no entanto, após a interrupção da gravidez, retomar o sentido de suas vidas. O sofrimento é realmente minimizado com o passar do tempo, ficam as lembranças dos momentos tristes e dificeis, mas o sofrimento deixa de existir com a elaboração do luto e de toda a vivência. Neste estudo a grande maioria não acredita em 'castigo' por terem optado pela interrupção da gestação.

Ressalta-se, no entanto, que, neste estudo, todas as pacientes foram acompanhadas por psicólogo no momento da opção pela interrupção da gravidez, os conteúdos inconscientes foram trazidos ao consciente e as pacientes puderam refletir sobre suas posições. É provável que a ausência de sentimento de culpa ou depressão pós-interrupção esteja diretamente relacionada a esse processo de intensa revisão de valores morais, culturais e melhor compreensão dos aspectos psíquicos em funcionamento. Dessa forma, fica claro que o acompanhamento psicológico é de fundamental importância no processo de decisão. Entretanto, estudos com entrevistas semi-estruturadas demonstram que é dificil estabelecer padrões do modo como devem ser oferecidas as informações sobre o acometimento fetal, reforçando a idéia da necessidade do atendimento multidisciplinar nesses $\operatorname{casos}^{14}$.

Após a interrupção da gravidez, ainda na sala de parto, os profissionais de saúde também devem se abster de emitir julgamentos. Mesmo em estudos com médicos residentes dos Estados Unidos, verifica-se que a taxa de participação em procedimentos de aborto diminui de forma significativa quando se reduz a gravidade da anomalia fetal ${ }^{23}$.

A participação da equipe que assiste à paciente no momento do parto deve procurar seguir condutas claras sobre os procedimentos a serem adotados. Em estudo realizado em seis hospitais da Califórnia, EUA, verifica-se que $77 \%$ das enfermeiras concordariam em prestar atendimento às pacientes internadas para interrupção da gestação por anomalia fetal letal ${ }^{24}$. Algumas mães necessitam visualizar o feto com todos os problemas, para que consigam crer plenamente no diagnóstico e estabelecer um sentido psíquico para essa vivência. Outras preferem não entrar em contato, por acreditar que isso dificultaria o luto. A escolha é pessoal e deve ser respeitada pelos profissionais que estão fazendo o atendimento da paciente, pois mesmo tendo optado pela interrupção, o casal não consegue conceber esse ato como eliminando a vida do filho. Entendem o processo como escolhendo o momento da perda, mas o vínculo afetivo com a "criança" se mantém; por isso, exigirão respeito e cuidado com o feto durante todo o processo.

É importante que o casal seja reavaliado após o processo de interrupção da gravidez para que se possam identificar os processos psíquicos deste momento, identificando se há necessidade de acompanhamento psicológico para auxiliar na elaboração do luto e da situação vivida.

Este trabalho possibilitou compreender os processos psíquicos vivenciados após a interrupção judicial da gestação por malformação fetal letal. Houve reconhecimento de que a interrupção da gravidez foi a melhor escolha, e que efetivamente minimizou o sofrimento. Não foram identificadas reações de arrependimento ou culpa. A participação ativa do casal no processo de decisão, com ampla reflexão sobre os valores morais e culturais e com a elucidação de aspectos inconscientes, é fundamental para minimizar os sentimentos negativos.

O presente estudo permite concluir que as angústias vivenciadas demonstram ser processo de reflexão, que é de fundamental importância para decisão consciente e posterior satisfação com a atitude tomada. $\mathrm{O}$ acompanhamento psicológico permite revisão dos valores morais e culturais para auxiliar a tomada de decisões visando minimizar o sofrimento vivido. 


\section{Referências}

1. Zugaib M, Okumura M. Ultra-sonografia obstétrica. In: Okumura M, Zugaib M, editores. Ultra-sonografia em obstetrícia. São Paulo: Sarvier; 2002. p.7-11

2. Marteau T, Drake H, Bobrow M. Counselling following diagnosis of a fetal abnormality: the differing approaches of obstetricians, clinical geneticists, and genetic nurses. J Med Genet. 1994;31(11):864-7.

3. Marteau TM, Dormandy E. Facilitating informed choice in prenatal testing: how well are we doing? Am J Med Genet. 2001;106(3):185-90.

4. Locock L, Crawford J, Crawford J. The parents' journey: continuing a pregnancy after a diagnosis of Patau's syndrome. BMJ. 2005;331(7526):1186-9.

5. Mansfield C, Hopfer S, Marteau TM. Termination rates after prenatal diagnosis of Down syndrome, spina bifida, anencephaly, and Turner and Klinefelter syndromes: a systematic literature review. European Concerted Action: DADA (Decision-making After the Diagnosis of a fetal Abnormality). Prenat Diagn. 1999;19(9):808-12.

6. Kowalcek I, Muhlhoff A, Bachmann S, Gembruch U. Depressive reactions and stress related to prenatal medicine procedures. Ultrasound Obstet Gynecol. 2002;19(1):18-23.

7. Davies V, Gledhill J, McFadyen A, Whitlow B, Economides D. Psychological outcome in women undergoing termination of pregnancy for ultrasounddetected fetal anomaly in the first and second trimesters: a pilot study. Ultrasound Obstet Gynecol. 2005;25(4):389-92.

8. Korenromp MJ, Christiaens GC, van den Bout J, Mulder EJ, Hunfeld JA, Bilardo CM, et al. Long-term psychological consequences of pregnancy termination for fetal abnormality: a cross-sectional study. Prenat Diagn. 2005;25(3):253-60.

9. van der Pal-de Bruin KM, Graafmans W, Biermans MC, Richardus JH, Zijlstra AG, Reefhuis J, et al. The influence of prenatal screening and termination of pregnancy on perinatal mortality rates. Prenat Diagn. 2002;22(11):966-72.

10. Decreto-Lei $\mathrm{n}^{\circ} 2.848$, de 7 de dezembro de 1940. Código penal. In: Delmanto C, Delmanto R, Delmanto Junior R, Delmanto FMA, editores. Código penal comentado. São Paulo: Renovar; 2002. p. 267-71.

11. Diniz D. Aborto e inviabilidad fetal: el debate brasileno. Cad Saude Publica. 2005;21(2):634-9.

12. Dallaire L, Lortie G, Des Rochers M, Clermont R, Vachon C. Parental reaction and adaptability to the prenatal diagnosis of fetal defect or genetic disease leading to pregnancy interruption. Prenat Diagn. 1995;15(3):249-59.

13. Burgoine GA, Van Kirk SD, Romm J, Edelman AB, Jacobson SL, Jensen JT. Comparison of perinatal grief after dilation and evacuation or labor induction in second trimester terminations for fetal anomalies. Am J Obstet Gynecol. 2005;192(6):1928-32.

14. Moutard ML, Moutel G, Francois I, Fauriel I, Feingold $\mathrm{J}$, Ponsot G, et al. Prenatal diagnosis of cerebral malformation with an uncertain prognosis: a study concerning couple's information and consequences on pregnancy. Ann Genet. 2004;47(1):41-51.

15. Statham H, Solomou W, Chitty L. Prenatal diagnosis of fetal abnormality: psychological effects on women in low-risk pregnancies. Baillieres Best Pract Res Clin Obstet Gynaecol. 2000;14(4):731-47.

16. Benute GR, Gollop TR. O que acontece com os casais após o diagnóstico de malformação fetal? Femina. 2002;30(9):661-3.

17. Duailibi R, Cabral ACV, Vitral ZN, Leite HV, Domont RJ. Acompanhamento psicológico de mães de fetos malformados no Centro de Medicina Fetal da Universidade Federal de Minas Gerais. Femina. 2003;31(1):27-30.

18. Ralston SJ, Wertz D, Chelmow D, Craigo SD, Bianchi DW. Pregnancy outcomes after prenatal diagnosis of aneuploidy. Obstet Gynecol. 2001;97(5 Pt 1):729-33.

19. Wertz DC, Fletcher JC. Feminist criticism of prenatal diagnosis: a response. Clin Obstet Gynecol. 1993;36(3):541-67.

20. Bell M, Stoneman Z. Reactions to prenatal testing: reflection of religiosity and attitudes toward abortion and people with disabilities. Am J Ment Retard. 2000; 105(1):1-13.

21. Garne E, Loane M, Dolk H, De Vigan C, Scarano G, Tucker D, et al. Prenatal diagnosis of severe structural congenital malformations in Europe. Ultrasound Obstet Gynecol. 2005;25(1):6-11.

22.Leung TN, Ching Chau MM, Chang JJ, Leung TY, Fung TY, Lau TK. Attitudes towards termination of pregnancy among Hong Kong Chinese women attending prenatal diagnosis counselling clinic. Prenat Diagn. 2004;24(7):546-51.

23. Fischer RL, Schaeffer K, Hunter RL. Attitudes of obstetrics and gynecology residents toward abortion participation: a Philadelphia area survey. Contraception. 2005;72(3):200-5.

24. Marek MJ. Nurses' attitudes toward pregnancy termination in the labor and delivery setting. $\mathrm{J}$ Obstet Gynecol Neonatal Nurs. 2004;33(4):472-9. 